\title{
Correction to: Unequal Opportunity: School and Neighborhood Segregation in the USA
}

\author{
Ann Owens ${ }^{1}$
}

(c) Springer Science+Business Media, LLC, part of Springer Nature 2020

Correction to: Race and Social Problems (2020) 12:29-41 https://doi.org/10.1007/s12552-019-09274-z

The original version of this article unfortunately contained an error. The author would like to correct the error with this erratum.

In the original publication of the article, Table 4 contained some errors. This erratum provides the corrected Table 4.

The original article can be found online at https://doi.org/10.1007/ s12552-019-09274-z.

$\triangle$ Ann Owens

annowens@usc.edu

1 University of Southern California, University Park Campus, MC 1059, Los Angeles, CA 90089-1059, USA 
Table 4 Characteristics of the average child's neighborhood, by race/ethnicity and poverty status, 2013-2017

\begin{tabular}{|c|c|c|c|c|c|c|c|c|}
\hline & \multicolumn{4}{|c|}{ Poor children } & \multicolumn{4}{|c|}{ Non-poor children } \\
\hline & Hispanic & Black & White & Asian & Hispanic & Black & White & Asian \\
\hline \multicolumn{9}{|l|}{ Racial/ethnic composition } \\
\hline Percent Hispanic & $53.9 \%$ & $15.7 \%$ & $10.4 \%$ & $23.2 \%$ & $43.9 \%$ & $15.8 \%$ & $9.3 \%$ & $17.2 \%$ \\
\hline Percent black & $11.1 \%$ & $48.7 \%$ & $7.9 \%$ & $11.6 \%$ & $9.5 \%$ & $40.5 \%$ & $6.1 \%$ & $8.0 \%$ \\
\hline Percent white & $28.6 \%$ & $30.1 \%$ & $76.1 \%$ & $39.2 \%$ & $38.0 \%$ & $36.6 \%$ & $77.9 \%$ & $48.4 \%$ \\
\hline Percent Asian & $4.0 \%$ & $2.7 \%$ & $2.5 \%$ & $21.7 \%$ & $5.9 \%$ & $4.2 \%$ & $3.9 \%$ & $22.4 \%$ \\
\hline Percent American Indian & $0.5 \%$ & $0.3 \%$ & $0.6 \%$ & $0.6 \%$ & $0.5 \%$ & $0.3 \%$ & $0.5 \%$ & $0.3 \%$ \\
\hline Percent other/multiracial & $1.9 \%$ & $2.4 \%$ & $2.4 \%$ & $3.7 \%$ & $2.2 \%$ & $2.6 \%$ & $2.3 \%$ & $3.6 \%$ \\
\hline \multicolumn{9}{|l|}{ Social composition } \\
\hline Percent foreign-born & $24.7 \%$ & $11.3 \%$ & $7.7 \%$ & $28.3 \%$ & $22.8 \%$ & $14.1 \%$ & $8.4 \%$ & $26.0 \%$ \\
\hline Percent single female-headed $\mathrm{HH}$ & $20.2 \%$ & $25.0 \%$ & $13.0 \%$ & $15.6 \%$ & $16.2 \%$ & $20.0 \%$ & $10.2 \%$ & $11.5 \%$ \\
\hline Percent $<$ high school & $27.4 \%$ & $19.5 \%$ & $14.0 \%$ & $18.4 \%$ & $20.6 \%$ & $14.8 \%$ & $9.1 \%$ & $10.6 \%$ \\
\hline Percent $\mathrm{BA}+$ & $17.6 \%$ & $18.4 \%$ & $22.7 \%$ & $29.2 \%$ & $24.2 \%$ & $25.6 \%$ & $34.3 \%$ & $43.1 \%$ \\
\hline \multicolumn{9}{|l|}{ Economic composition } \\
\hline Median HH income & $\$ 43,230$ & $\$ 38,346$ & $\$ 52,419$ & $\$ 59,315$ & $\$ 59,165$ & $\$ 54,464$ & $\$ 73,799$ & $\$ 88,036$ \\
\hline Percent in labor force & $61.4 \%$ & $60.2 \%$ & $60.8 \%$ & $64.2 \%$ & $64.7 \%$ & $64.5 \%$ & $64.8 \%$ & $66.9 \%$ \\
\hline Poverty rate & $27.8 \%$ & $30.2 \%$ & $18.6 \%$ & $20.4 \%$ & $17.2 \%$ & $18.5 \%$ & $10.4 \%$ & $10.1 \%$ \\
\hline Percent public assistance & $4.8 \%$ & $4.9 \%$ & $3.0 \%$ & $4.3 \%$ & $3.4 \%$ & $3.4 \%$ & $1.9 \%$ & $2.4 \%$ \\
\hline \multicolumn{9}{|l|}{ Housing characteristics } \\
\hline Vacancy rate & $10.7 \%$ & $14.4 \%$ & $12.5 \%$ & $8.3 \%$ & $8.8 \%$ & $11.0 \%$ & $9.5 \%$ & $6.4 \%$ \\
\hline Renter rate & $49.8 \%$ & $53.0 \%$ & $34.3 \%$ & $49.3 \%$ & $41.5 \%$ & $43.8 \%$ & $26.7 \%$ & $36.1 \%$ \\
\hline
\end{tabular}

Data on race by Hispanic ethnicity by age is only available for whites. Therefore, the "white" columns refer to non-Hispanic children, but black and Asian include those that also identify as Hispanic. Data on race by Hispanic ethnicity for allpersons is available for all groups, so the black, white, Asian, American Indian, and other/multiracial rows under"racial/ethnic composition" are non-Hispanic

Publisher's Note Springer Nature remains neutral with regard to jurisdictional claims in published maps and institutional affiliations. 